\title{
ROBUSTNESS OF SURROGATES OF BIODIVERSITY IN MARINE BENTHIC COMMUNITIES
}

\author{
Regina H. Magierowski and Craig R. Johnson ${ }^{1}$ \\ School of Zoology and Tasmanian Aquaculture and Fisheries Institute, University of Tasmania, Private Bag 05, \\ Hobart, Tasmania 7001 Australia
}

\begin{abstract}
The usefulness of surrogates to estimate complex variables describing community structure, such as the various components of biodiversity, is long established. Most attention has been given to surrogates of species richness and species diversity and has focused on identifying a subset of taxa as a surrogate of total community richness or diversity. In adopting a surrogate measure, it is assumed that the relationship between the surrogate(s) and total richness or diversity is consistent in both space and time. These assumptions are rarely examined explicitly. We examined the robustness of potential surrogates of familial richness and multivariate community structure for macrofauna communities inhabiting artificial kelp holdfasts by comparing among communities of dissimilar ages and among communities established at different times of the year. This is important because most benthic "landscapes" will be a mosaic of patches reflecting different intensities, frequencies, and timing of disturbances. The total abundance of organisms and familial richness of crustaceans or polychaetes were all good predictors of total familial richness $\left(R^{2}>0.68\right)$. In contrast, while the familial richness of other groups, such as mollusks and echinoderms, were well correlated with total familial richness for communities at an early stage of development, the strength of these relationships declined with community age. For multivariate community structure, carefully selected subsets of $\sim 10 \%$ of the total taxa yielded similar patterns to the total suite of taxa, irrespective of the age of the community. Thus, useful surrogates of both familial richness and multivariate community structure can be identified for this type of community. However, the choice of technique for selecting surrogate taxa largely depends on the nature of the pilot data available, and careful selection is required to ensure that surrogates perform consistently across different-aged communities. While the specific taxa selected as surrogates will vary among different communities, and possibly even among similar communities at different sites, the techniques and the concepts we address are applicable to any community type.
\end{abstract}

Key words: biodiversity surrogates; diversity; Ecklonia radiata; kelp holdfasts; macrofauna; multivariate analyses; southern Australia; temporal variation; richness.

\section{INTRODUCTION}

Biodiversity is an important and fundamental concept in ecology, but it is also highly complex because it encompasses functional system components in addition to structural components such as genetic, species, habitat, and ecosystem diversity (Franklin 1988, Noss 1990, Vane-Wright et al. 1991). Accordingly, the assessment of biodiversity usually relies on a proxy, such as species richness or diversity. However, for conservation biologists, even attempting to enumerate all species is time-consuming, labor-intensive, and a task requiring expertise (Daily and Ehrlich 1995, Kitching et al. 2001), so that extrapolative and other techniques will always be sought to optimize efficiencies. This is particularly true while taxonomy continues to be a low

Manuscript received 22 November 2005; revised 4 April 2006; accepted 5 April 2006. Corresponding Editor: P. K. Dayton.

${ }^{1}$ Corresponding author.

E-mail: craig.johnson@utas.edu.au priority for both researchers and funding bodies (Valdecasas and Camacho 2003, Wheeler et al. 2004) and is conducted in relative isolation from the other disciplines in the life sciences (Dayrat 2005).

Research has long established the potential usefulness of one type of extrapolative approach, namely the use of various indices to estimate and monitor ecological impact and describe ecosystem integrity (Noss 1990). These indices are known as biological indicators or "bioindicators" and their application has been widespread across terrestrial (e.g., Kremen 1992, Samways and Steytler 1996, Warman et al. 2004), freshwater (e.g., Karr 1981, Savage 1982, Fore et al. 1996), and marine environments (e.g., Olsgard et al. 2003).

Of several different kinds of indices, each with their relative merits (see Noss 1990, McGeoch 1998), surrogates for biodiversity are among the most commonly used (McGeoch 1998). This approach usually involves selecting a subset of species (or higher taxon) from a community as a surrogate of either the biodiversity of another taxon or as a surrogate of the total biodiversity 
of a community. In these studies, the aim is usually to monitor or compare ecosystem properties (such as biodiversity) or to detect environmental impact (e.g., Karr et al. 1987, Gaston and Williams 1993, Gaston and Blackburn 1995, Gaston 1996b). Taxa may be selected because they are abundant or are known to be sensitive to particular environmental perturbations, or because of a predilection toward particular taxa, which may simply reflect available taxonomic expertise.

In the case of monitoring taxa thought to be sensitive to environmental impact, considerable effort, particularly in freshwater systems, has been given to ratifying indicators of impact and testing underlying assumptions, (e.g., Karr et al. 1987, Barbour et al. 1992, Diamond et al. 1996, Fore et al. 1996). However, this is not the case in the use of surrogates for more general monitoring of biodiversity, where in only a few cases attention has been given to comparing the performance of a range of potential choices of surrogates (e.g., Oliver and Beattie 1996, Lund and Rahbek 2002, Anderson et al. 2005b) or to the underlying assumptions (McGeoch 1998). The key assumption made in attempting to identify and apply surrogates of total biodiversity is that the relationship between the selected surrogate taxa and total biodiversity is constant in space and time (Colwell and Coddington 1994). Spatial consistency in the identity of surrogates has been examined and, at least in some cases, it is clear that surrogates should not be applied across regional spatial scales because species relationships are not necessarily the same in different regions (Beccaloni and Gaston 1995, Gaston 1996a, b, Anderson et al. 2005b). Surprisingly however, the assumption that within any particular location the relationship between the surrogate and total biodiversity is constant through time (e.g., Kitching et al. 2000) has received scant attention. Lack of discussion of this important aspect of the application of surrogacy arguably reflects that the majority of work employing surrogates has focused on comparing biodiversity among sites rather than through time, even though samples are usually pooled across several sampling periods (e.g., Pearson and Cassola 1992, Beccaloni and Gaston 1995, Gaston and Blackburn 1995, Erdmann and Caldwell 1997, Garson et al. 2002, Lund and Rahbek 2002, Olsgard et al. 2003, Warman et al. 2004). It is usually assumed (often implicitly) that differences in biodiversity indicated from samples collected at different sites and at different times are due to spatial variability. It is therefore assumed that surrogates are temporally consistent.

It is well recognized that community structure may vary temporally depending on successional status (i.e., disturbance history; e.g., Dean and Connell 1987) and seasonal effects. Moreover, these two factors may interact such that the relative abundance of a taxon for a given successional state may depend on the timing of the original disturbance (Dayton et al. 1984, Chapman and Johnson 1990, Underwood and Anderson
1994, Nandakumar 1996). While changes in the relative abundance of a suite of taxa does not necessarily infer poor suitability as a surrogate of total biodiversity, it is nonetheless important to establish robustness in the performance of putative surrogates in the face of temporal change in community structure. Thus, suitable surrogates for monitoring biodiversity are those that correlate with changes in biodiversity, whether due to spatial variability, succession, season, or disturbance (Colwell and Coddington 1994). Note that, in this context, the assessment of the performance of surrogates in monitoring biodiversity differs from the traditional approach used to assess surrogates of environmental impact. Good surrogates of environmental impact are relatively static through space and time across reference sites, but sensitive to impact levels (Karr et al. 1987, Underwood and Peterson 1988, Barbour et al. 1992, Glasby and Underwood 1998). Thus, surrogates intended to detect specific environmental impacts (often based on sensitive or intolerant taxa or functional groups; e.g., Barbour et al. 1992) will not necessarily be useful as surrogates to monitor or compare total biodiversity within or between sites. Moreover, surrogates of total diversity are more likely to be based on the species or familial richness of a single higher-level taxon or the abundance of several taxa (Hammond 1994).

In this paper we explore whether temporal variability is an important consideration in the selection of a structural biodiversity surrogate by examining the effects on surrogate performance of community age and the season of sampling, two distinct components of temporal variability in community structure. By doing so, we also explore several potentially useful techniques to define surrogates and compare their performance. We use subtidal marine communities inhabiting artificial kelp holdfasts as a model system, and given that biodiversity can be defined validly in a number of ways (Noss 1990), we examine both univariate and multivariate indices of familial biodiversity. We show that temporally stable surrogates can be identified for communities of different ages and across different seasons of initial deployment and final assessment. However, exactly which taxa to use depends on the particular community in question. Moreover, the choice of which technique to use for selecting surrogate taxa depends largely on the nature of the reference data available.

\section{Methods \\ Experimental design and fieldwork}

Artificial kelp holdfasts (hereafter "holdfasts") were constructed of bundles of 10 150-mm lengths of polypropylene rope bound together at one end with a plastic cable tie. At the other end, the rope strands were separated, splayed, and glued to a $100 \times 100 \mathrm{~mm}$ PVC base. The design was similar in size and physical complexity to the holdfasts of Ecklonia radiata, the most common species of kelp in southern Australia. An 


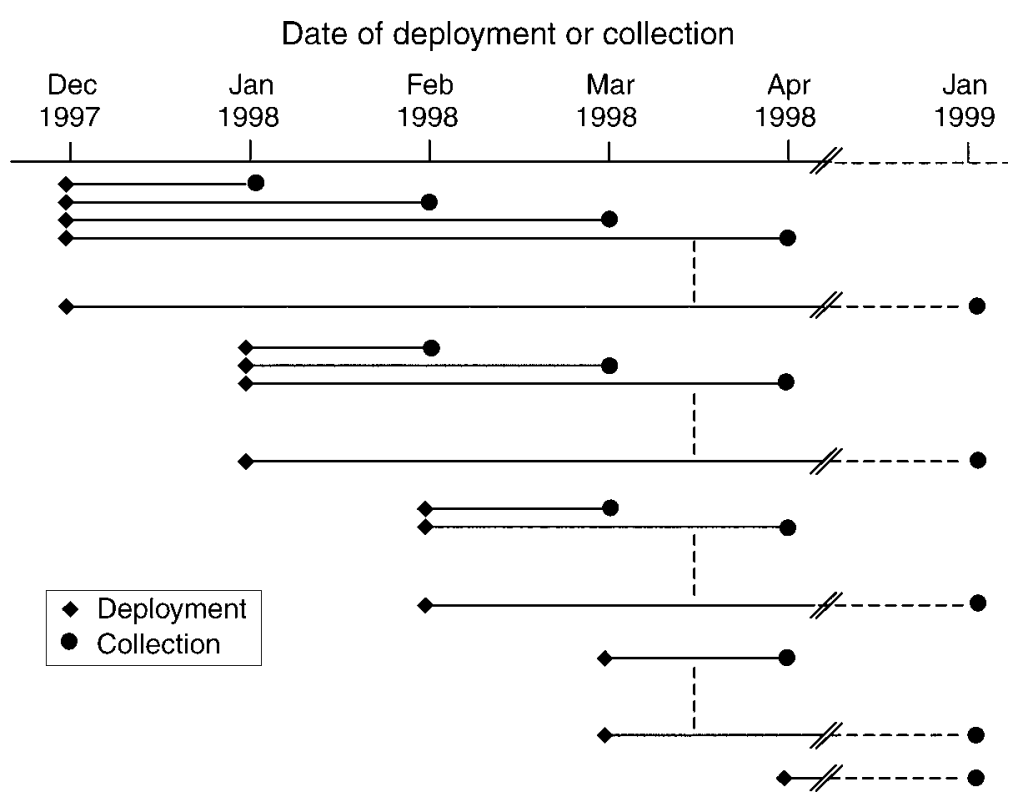

FIG. 1. Schematic representation of the sequence of deployment and collection of artificial kelp holdfasts. The entire experiment extended from December 1997 to January 1999. The complete design included 91 different treatments, each with unique deployment and collection dates, and there were six replicate holdfasts of each deployment/collection combination. Note however, that because of poor weather, we were unable to deploy and retrieve holdfasts exactly to this design (see Methods). Each line represents deployment and collection dates of six replicate holdfasts. Dashed lines show where treatments have been excluded for clarity.

earlier pilot study showed that this design developed a community similar to that found in E. radiata holdfasts from the same area.

Holdfasts were attached to concrete bricks with cable ties and distributed over sand adjacent to a reef at $\sim 8 \mathrm{~m}$ depth in the Derwent River Estuary, Tasmania, Australia $\left(42^{\circ} 57.7^{\prime} \mathrm{S}, 147^{\circ} 20.5^{\prime} \mathrm{E}\right)$. The study site was $100 \times 4 \mathrm{~m}$, and holdfasts were deployed randomly to 1 $\mathrm{m}$ grid coordinates. The rocky reef ran the full length $(100 \mathrm{~m})$ of the grid, and was likely the major source of immigrants to the holdfasts.

Holdfasts were deployed each month for 13 months beginning in December 1997. At each deployment, sufficient holdfasts were established to collect six replicate holdfasts each subsequent month until January 1999 (Fig. 1). An earlier pilot study indicated that six holdfasts was the minimum sampling intensity to adequately estimate natural variability across replicate holdfast communities. Deployment and collection dates were toward the end of the nominated month, weather permitting. Water temperature peaked in January and February at $18^{\circ} \mathrm{C}$ and reached a minimum of $11^{\circ} \mathrm{C}$ during June, July, and August. A total of 408 holdfasts were deployed to random positions on the grid and later recovered.

Monthly collections involved recovering six randomly selected, replicate holdfasts from each previous month of deployment. Holdfasts were gently covered with a plastic bag before cutting the cable ties attaching the holdfast to its concrete brick and sealing the bag for transport to the surface. Vacated grid positions were open to subsequent deployment of another artificial holdfast (if randomly selected). Due to poor weather, holdfasts could not be collected or deployed in September and collections were not possible in June. For treatments deployed in December 1997, four replicates (rather than six) were collected each subsequent month.

Holdfasts were preserved in $\sim 5 \%$ buffered formalin. For processing, holdfasts were broken open and washed thoroughly over a $1-\mathrm{mm}$ sieve to remove all animals. Solitary animals retained on the sieve were identified where possible to the level of family, the most notable exception being amphipods, which were identified to suborder. We deemed taxonomic resolution to the level of family as the optimal cost-benefit trade-off of research time to information gain, given the large abundance of organisms encountered (148 841 individuals), and that family-level patterns typically reflect patterns at the species level (Williams and Gaston 1994, Faith et al. 1995, James et al. 1995, Somerfield and Clarke 1995, Balmford et al. 1996, Olsgard et al. 1997, Mistri and Rossi 2001, Dahl and Dahl 2002, Olsgard et al. 2003, Anderson et al. 2005a, b).

\section{Surrogacy analysis: univariate surrogates of community richness}

Higher-level taxa likely to prove suitable surrogates are those that are proportionally abundant and rich. In our communities the Crustacea, Mollusca, Polychaeta, 
and Echinodermata taxa were all potential candidates. Polychaetes (Olsgard and Somerfield 2000, Olsgard et al. 2003) and mollusks (Gladstone 2002) in particular have been suggested as possible surrogates of marine macrofauna communities in soft sediment and on rocky shores, respectively, because they are both abundant and ecologically important groups. The total abundance of all fauna counted within a holdfast was also used because it is the easiest of all community properties to enumerate. Thus, any surrogate taxon selected must be able to outperform total abundance as a surrogate to be cost-effective.

The average familial richness (by deployment date and community age) of each taxonomic group and the average total abundance of all organisms were recorded and plotted against the average total familial richness (averages were of each group of six replicate holdfasts). Regression analysis was used to compare the goodness of fit $\left(R^{2}\right)$ for each potential surrogate. Good surrogate taxa will have a high $R^{2}$ value, reflecting a less noisy, and thus more predictable, relationship with richness. While we acknowledge that the familial richness of each taxonomic group is not independent of total familial richness and that this may be problematic in regression analysis (Schulze et al. 2004), we proceeded with the analysis in this way simply because the focus of the work was to evaluate how well the richness of a specific taxon was correlated with total richness.

If surrogate taxa are robust, then the relationship between each potential surrogate and total richness should not change with community age or deployment date. This premise was examined using ANCOVA. Because of evidence of a correlation between community age and richness (at least for colder months), regression analysis was also used to determine whether the goodness of fit (adjusted $R^{2}$ ) of the relationship between richness of particular taxa and total richness varied with community age. Adjusted $R^{2}$ was used because the degrees of freedom varied for the various community age categories.

\section{Surrogacy analysis: multivariate surrogates of community pattern}

We compared several approaches to select subsets of families that may reflect the multivariate patterns seen in the complete kelp holdfast communities in the experiment. The first was a taxonomic approach, where all families within a higher taxon (usually phylum) were selected. We also used two techniques within the PRIMER5 statistical software package to select subsets of families, namely BVSTEP (Clarke and Warwick 1998, 2001) and SIMPER (Clarke and Warwick 2001). Surrogates selected using these techniques were compared with surrogates defined by random selections of taxa and selections of the numerically abundant taxa. The performance of each subset of surrogates was tested by comparing the matrices of similarities between all community ages and deployment dates for each surrogate set with the equivalent similarity matrix determined from the complete faunal complement.

Taxonomic approach.-The four higher taxa selected were the same groups used to assess "univariate surrogates" (Mollusca, Crustacea, Polychaeta, and Echinodermata). All families within each of these groups were identified, and each higher group was considered separately in contrasting the "treatments" of community age and deployment date. Thus, a similarity matrix describing the similarity between each pair of treatments was produced for each of the higher taxa.

SIMPER.-SIMPER is used to identify those taxa that either contribute most to the average similarity within a treatment (i.e., between replicates) or to the average dissimilarity between two treatments (Clarke and Warwick 2001). We used SIMPER to identify families that contributed most to the average similarity among replicate communities of a particular deployment date. Since a suitable surrogate family must also occur consistently within holdfasts, the ratio of the average similarity to its standard deviation (SIM/sD) for each family (see Clarke and Warwick 2001) was also used to select families. Data were first transformed using a fourth-root transformation. For each deployment date selected as a reference point (arbitrarily January-April 1998), results of within-group average similarities were presented for each community age. Families were included in the surrogate set for a particular deployment date if they were selected for at least one community age. The number of families included within a surrogate set generated by SIMPER was therefore variable depending on deployment dates.

Because SIMPER was used to look for similarities within treatments rather than among treatments (as it is only able to make pairwise comparisons between treatments), we also tested whether the results from the SIMPER analyses were consistent regardless of which deployment date or community age was selected. This was done by identifying the surrogate set for each treatment combination of deployment date and community age and comparing these surrogate sets using the Bray-Curtis similarity measure (Bray and Curtis 1957). Surrogate sets were compared across community ages for a given deployment date and across deployment dates for a given community age. Each surrogate set was compared to a reference point (either the first deployment date or first community age) to look for directional deviation from that point. A directional decline in BrayCurtis similarity would suggest that the surrogate set identified by SIMPER indicated a gradual and directional change in community composition. Looking for a directional change is important because the Bray-Curtis measure is of overall similarity, not absolute community structure (i.e., the community structure of two samples of identical Bray-Curtis similarity to a given reference community may be dissimilar). Uniformly low BrayCurtis values would suggest that there was no consistency in families selected by SIMPER across deploy- 
ment dates or community ages. Note that we also conducted these analyses using a different arbitrary reference point (viz., the average surrogate subset), but since the interpretation of the results was similar, this analysis is not presented.

BVSTEP.-BVSTEP is a stepwise algorithm used most frequently to identify environmental variables that best correlate with patterns in biological data (Clarke and Warwick 2001). The routine identifies combinations of environmental variables that yield the highest rank correlation between similarity matrices based on biological and environmental variables (where similarity matrices describe similarities among samples). In a similar approach, if the original data matrix of biological variables is used instead of the environmental variables, BVSTEP can be used to exclude taxa that are redundant in explaining community level patterns (Clarke and Warwick 1998).

We used BVSTEP to identify potential surrogate sets of families (regardless of which higher taxon they came from), which best reflected community level patterns through time for each of four deployment dates (January-April 1998). Early deployment dates were selected because they yielded longer time series in community development. December 1997 was not used because there were fewer replicates for this month. Similarity matrices describing Bray-Curtis similarity among communities of different ages but identical deployment date were based on fourth-root-transformed data. Selections of taxa in defining surrogate sets were based on highest values of the weighted or harmonic Spearman rank correlation coefficient, $\rho_{\mathrm{w}}$ (Clarke and Ainsworth 1993). We selected the best results presented by BVSTEP for combinations of 5, 10, 12, 15, and 20 families. Thus, we obtained surrogate sets generated by BVSTEP for four different deployment dates, and for each of these deployment dates we had surrogate sets of $5,10,12,15$, and 20 families. Note that the total number of families detected across all holdfasts combined was exactly 100 .

Comparing multivariate surrogate sets.-We then examined whether the "optimal" surrogate sets identified by a taxonomic approach, SIMPER, and BVSTEP performed any better than combinations of 5, 10, 12, 15, and 20 families selected either randomly or on the basis of greatest total abundance. If the putative optimal surrogate sets are to be useful, they must perform better (achieve a higher $\left(\rho_{\mathrm{w}}\right)$ than selection by random choice or on the basis of abundance, which are both quicker and easier ways of selecting surrogate taxa.

Performance was assessed by how well each multivariate surrogate set predicted the multivariate relationships across all treatments of deployment date and community age, based on the complete suite of families. This was accomplished using "a second stage analysis" (Somerfield and Clarke 1995) in which similarity matrices (describing similarities between treatments of deployment date and community age) based on surro- gate sets were correlated with the equivalent similarity matrix based on all families (Anderson et al. 2005b). Correlations were calculated for each comparison using the weighted Spearman rank correlation coefficient (note that qualitative patterns in correlations were similar to those using the Spearman or Kendall correlation coefficients). Results of the second stage analysis can be presented using a nonmetric multidimensional scaling (NMDS) plot to display patterns in the degree of correlation (Somerfield and Clarke 1995, Anderson et al. 2005a,b). However, since an NMDS plot is only a two-dimensional estimation of all relationships (including those between surrogates), we present correlations between each surrogate set and the complete suite of families in table form.

Similarity matrices based on surrogate families that are highly correlated with the similarity matrix using all families should produce similar patterns in NMDS plots. Accordingly, NMDS plots were generated for some results to demonstrate how patterns changed as correlations between similarity matrices, based on the full data set and those based on subsets of species, declined. Data from March 1998 (which gave the best and worst correlations depending on the number of families selected), were used in this analysis. Note that some treatments were deleted from displayed plots to clarify presentation, but they were not deleted from the actual analysis.

\section{RESUlts}

\section{Univariate surrogates of community richness. surrogates by taxonomic group}

The relationship between each taxonomic group's average familial richness and average total familial richness was linear, while the relationship between average total abundance of all individuals and average familial richness was a power curve (Fig. 2). Surprisingly, the best surrogate of average total familial richness (highest $R^{2}$ ) was the average total abundance of all individuals $\left(R^{2}=0.85\right.$; Fig. 2$)$, followed closely by the familial richness of the Crustacea $\left(R^{2}=0.81\right)$, while the goodness of fit for the familial richness of the Mollusca, Polychaeta, and Echinodermata nonetheless indicated clear relationships $\left(R^{2}=0.62,0.68,0.57\right.$, respectively; Fig. 2). The observed correlations appear to be independent of the richness and total abundance of each taxon, since the number of families identified over the entire study period for Crustacea, Mollusca, Polychaeta, and Echinodermata was 24, 32, 21, and 12, respectively, and the number of individuals was 45448 , 21 636, 34 641, and 44319, respectively.

The slope of the relationship between average surrogate familial richness and average total familial richness did not vary significantly with community age or deployment date (Table 1). In contrast, however, the goodness of fit of these relationships did depend on community age and/or deployment date for some surrogate taxa (Fig. 3). While polychaetes showed a 

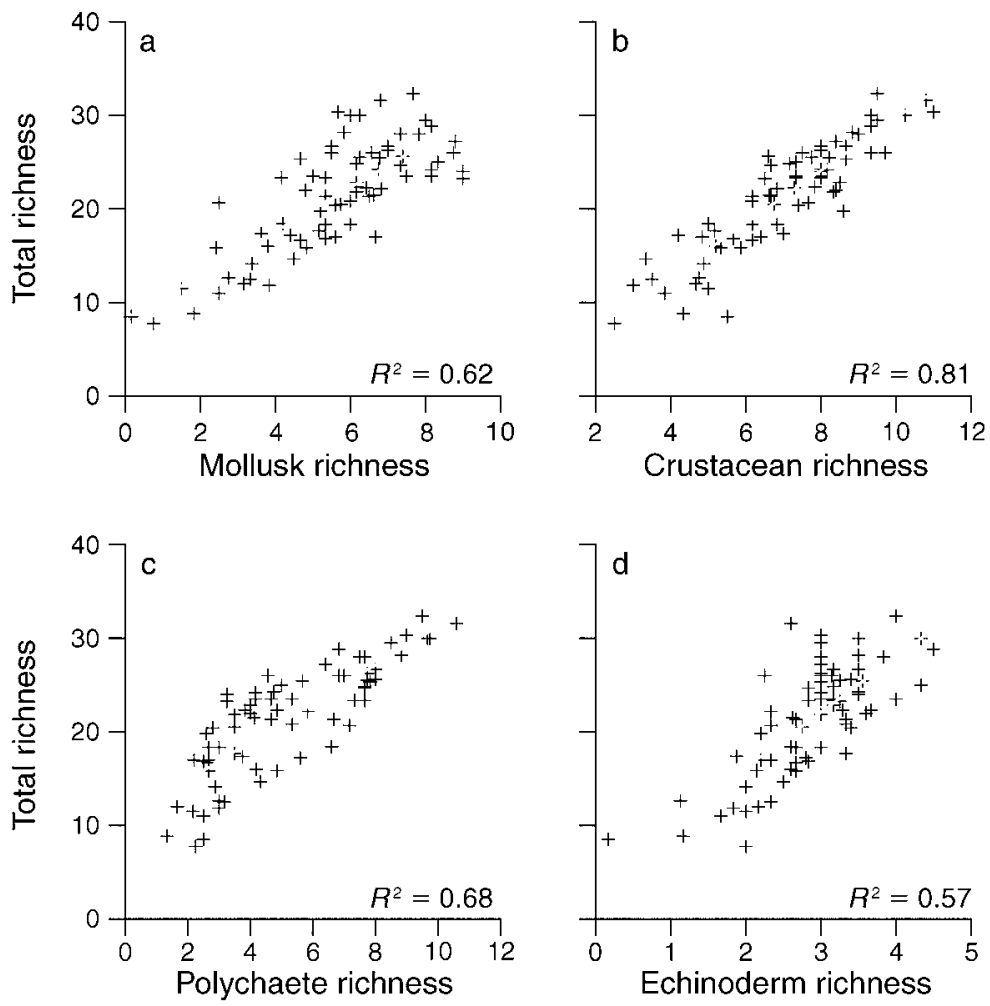

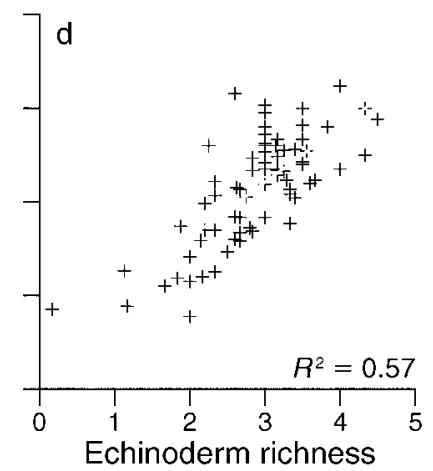

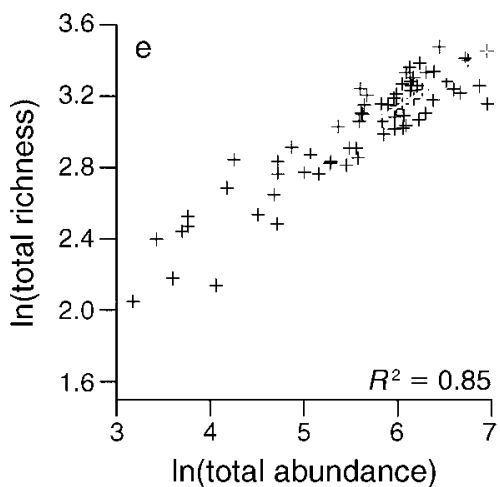

FIG. 2. (a-d) Relationship between mean number of taxa (mean total familial richness) and mean number of families in higher taxonomic groups and (e) mean total abundance (means are of six replicate holdfasts for each combination of deployment date $\times$ community age): (a) mollusk richness, $y=2.35 x+8.22$; (b) crustacean richness, $y=2.81 x+1.60$; (c) polychaete richness, $y=2.05 x+$ 10.72; (d) echinoderm richness, $y=5.92 x+4.40$; and (e) total abundance, $\ln (y)=0.33 \ln (x)+1.18$. All regression analyses were significant at $P<0.0001$.

consistently good fit irrespective of community age, mollusks and echinoderms showed a linear decline in goodness of fit with increasing community age (for mollusks, slope $=-0.67$, intercept $=1.13, P<0.0005, R^{2}$ $=0.91$; for echinoderms, slope $=-0.09$, intercept $=0.57$,

TABLE 1. Results of ANCOVAs showing that the slope of the relationship between average total richness and average richness of each higher taxon and between average total richness and average total abundance did not vary significantly with community age or date of deployment.

\begin{tabular}{lccc}
\hline \hline \multicolumn{1}{c}{ Test } & df & $F$ & $P$ \\
\hline Test of surrogate $\times$ community age & & \\
Mollusca & 10,47 & 1.93 & 0.07 \\
Crustacea & 10,47 & 0.87 & 0.57 \\
Polychaeta & 10,47 & 1.64 & 0.12 \\
Echinodermata & 10,47 & 0.59 & 0.81 \\
ln(total abundance) & 10,47 & 0.59 & 0.81 \\
Test of surrogate $\times$ deployment date & & \\
Mollusca & 10,48 & 1.41 & 0.20 \\
Crustacea & 10,48 & 1.17 & 0.33 \\
Polychaeta & 10,48 & 0.74 & 0.69 \\
Echinodermata & 10,48 & 1.07 & 0.40 \\
ln(total abundance) & 10,48 & 0.78 & 0.65 \\
\hline
\end{tabular}

Note: Significant differences in these relationships among community ages or among dates of deployment would be indicated by a significant interaction $(P<0.05)$ between the surrogate and community age/deployment date.
$P=0.005, R^{2}=0.59$ ). Thus, for these two phyla, the variability around the relationship depicted in Fig. 2 increased as the community aged. The overall trend for Crustacea and for the total abundance of all individuals appeared worse for communities of intermediate age, although there are too few data points to be certain of this trend.

\section{Multivariate surrogates of community richness}

Testing the robustness of SIMPER as a technique for selecting surrogate taxa.-For each deployment date there was no consistent evidence of a directional decline in similarity with community age that could be linked to a temporal shift in the identity of the surrogate subset (Fig. 4). However, for young communities $<5$ months old, there was some evidence of directional decline in similarity for the different deployment dates, suggesting a seasonal and/or successional influence in the identity of the surrogate subset (Fig. 5). By corollary, families typical of a set of holdfasts are less influenced by the season of deployment when communities are allowed to develop for a longer time period. Not surprisingly, by selecting a larger number of families within the surrogate set (by changing the cut off for including taxa), sensitivity to the season of deployment was usually 


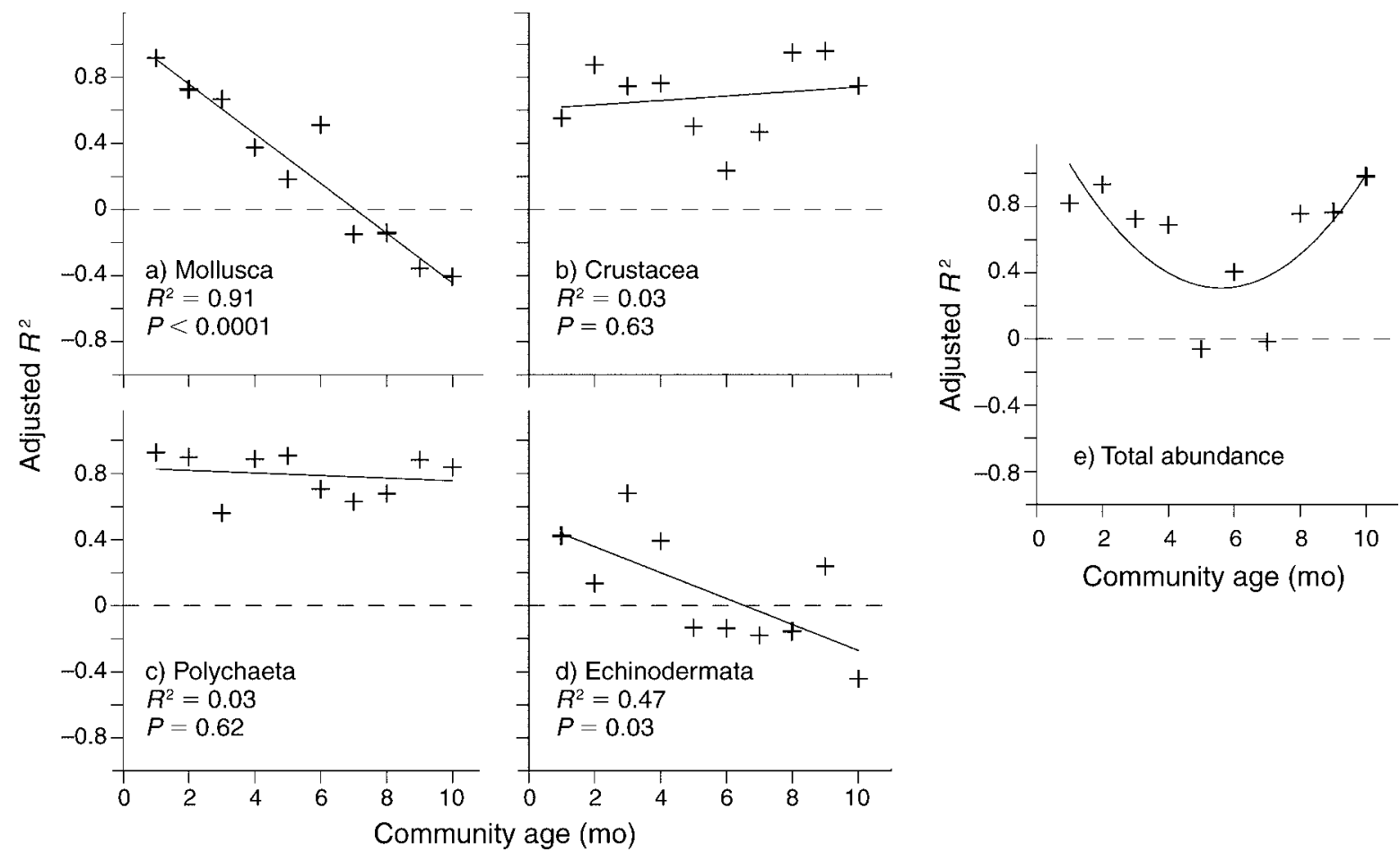

FIG. 3. Change in the goodness of fit (adjusted $R^{2}$ ) in the relationship between mean number of taxa (mean total familial richness) and the surrogate with age of the community. Surrogates are: (a) molluscan familial richness, $y=-0.15 x+1.06$; (b) crustacean familial richness, $y=0.01 x+0.61$; (c) polychaete familial richness, $y=-0.01 x+0.84$; (d) echinoderm familial richness, $y$ $=-0.08 x+0.5$; and (e) total abundance. Note that we used a regression technique able to calculate negative adjusted $R^{2}$ values.

reduced, and in most cases, low values of similarity between a given community and the reference point increased in magnitude (Fig. 5).

Comparison of multivariate surrogate communities.Predictably, the more families that occurred in a surrogate set, the better the performance in matching the patterns evident in the complete data set containing all 100 families (Table 2). For a given number of families, BVSTEP tended to perform as well or better than a surrogate set selected on the basis of most abundant families, while SIMPER only did as well as selecting the most abundant families (Table 2). For a given number of families, both techniques were better than selecting families randomly, and there was no noticeable effect of the deployment date selected (Table 2). Basing surrogate sets on a single higher taxon did not improve performance over random selection of families, even when the number of families was high (e.g., 32 mollusk families identified; Table 2). Of the higher taxa examined, patterns among treatments based on Crustacea best reflected patterns indicated by the full suite of families, even though this group contained fewer families than the mollusks (Table 2).

Results based on all families suggest an interaction between deployment date and community age (Fig. 6a). Older communities, particularly those $\geq 6$ months old, tended to cluster in a cloud. Younger communities (1 month) separated from this cloud, especially those developed in holdfasts deployed in cooler months. This suggests that succession towards a mature community occurs more quickly in the summer. This pattern becomes less clear as surrogate communities with progressively lower $\rho_{\mathrm{w}}$ values are selected, and is not readily discernable with a correlation less than $\rho_{\mathrm{w}}=$ 87.05 (Fig. 6b-f). By this criterion, it corresponds to identifying at least $10-15$ families (i.e., $10-15 \%$ of the total number of families detected). Results are presented for BVSTEP; however, using SIMPER to select surrogate sets, or simply basing the selection on the most abundant families, also produced similar results since $\rho_{\mathrm{w}}$ values of 91.33 and 90.54 were obtained for a selection of 14 families by SIMPER and a selection of 12 families on the basis of abundance respectively (Table 2).

\section{DisCUSSION}

\section{Univariate surrogates of community richness}

Biodiversity is often presented as the total species (or familial) richness at a site or time (Fleishman et al. 2004). Because total richness itself can be difficult to measure, researchers have suggested the use of surrogates based on the richness of particular higher taxa (e.g., Pearson and Cassola 1992, Beccaloni and Gaston 1995, Gaston and Blackburn 1995, Garson et al. 2002, Lund and Rahbek 2002, Olsgard et al. 2003, Warman et al. 2004). While the familial richness of crustaceans, 


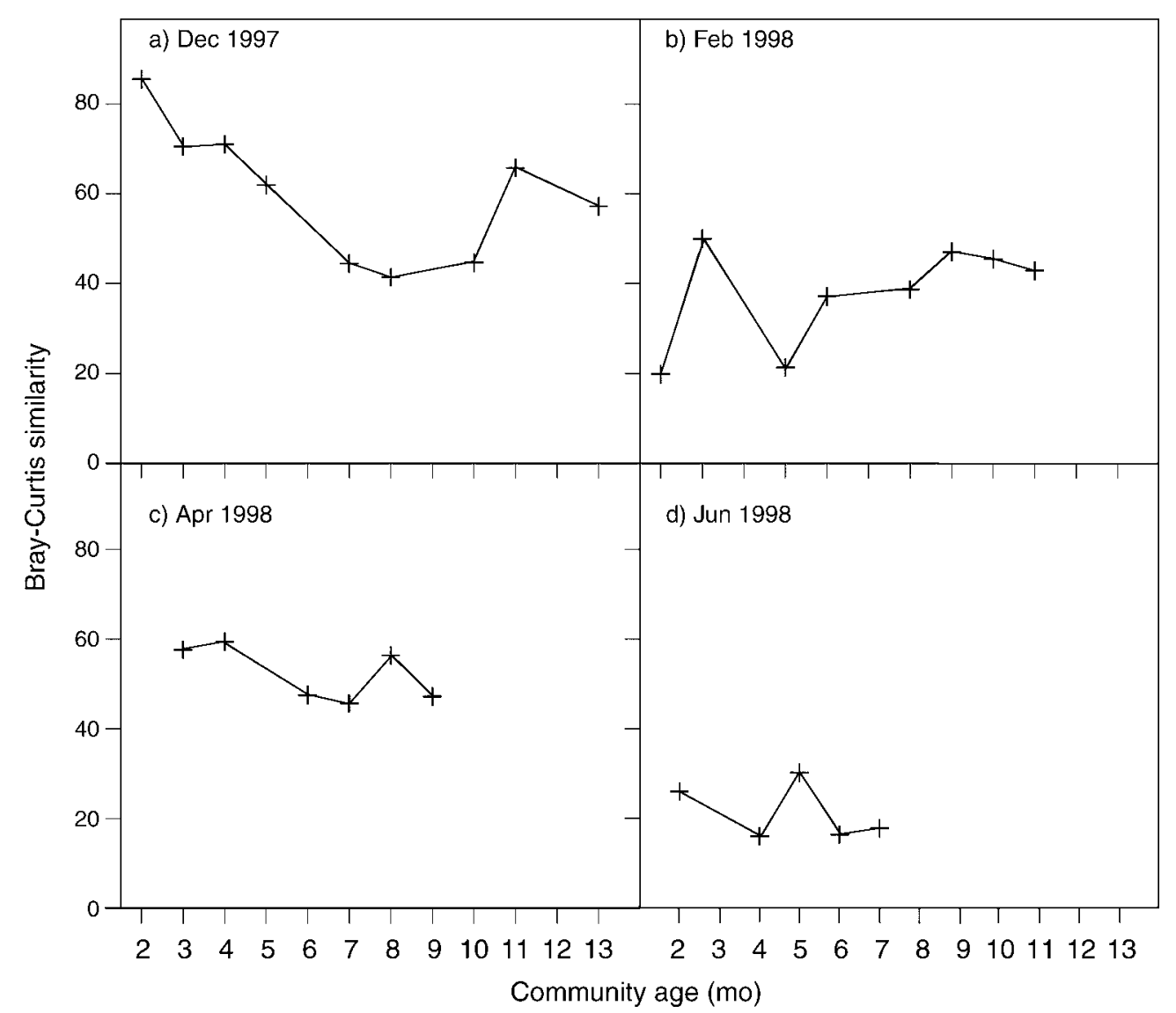

FIg. 4. Bray-Curtis similarity between the surrogate set identified by SIMPER (PRIMER5) for each community age and the surrogate set identified by SIMPER for one-month holdfasts for several representative deployment dates (see Appendix A for all deployment dates). Taxa were included in a surrogate set if they contributed $\geq 5 \%$ to the total variation and had a ratio of mean to standard deviation $\geq 1.4$. Deployment dates were (a) December 1997, (b) February 1998, (c) April 1998, and (d) June 1998.

mollusks, polychaetes, and echinoderms were all clearly correlated with total family richness in the artificial holdfasts, the best overall predictor of total richness was simply the total abundance of all individuals, irrespective of taxonomic affinity. However, the correlation between a surrogate and total familial richness was not indicative of the temporal consistency in performance as a surrogate. The Polychaeta, which did not achieve the highest correlation with total familial richness, was the most consistent univariate surrogate of total familial richness across communities of a range of ages. The familial richness and total abundance of a given taxonomic group were not good predictors of its performance with respect to the overall correlation with total familial richness and the temporal consistency of this correlation. This was also true in the multivariate analysis. These observations raise the possibility that the variety of ecological and/or functional roles, rather than the number of individuals or taxa within a taxon, may be a better determinant of the suitability of a surrogate set. These results are similar to those of Anderson et al. (2005b), who also found that high taxon richness was not highly correlated with a surrogate's ability to predict biodiversity in macrofauna communities in natural kelp holdfasts across different spatial scales in northeastern New Zealand (although total abundance may have been).

\section{Multivariate surrogates of family-level community pattern}

Since there are robust arguments to advocate use of multivariate similarity measures, such as Bray-Curtis to compare biodiversity among sites (and times) rather than total species richness or Shannon-Wiener diversity (e.g., Cao et al. 1996, Su et al. 2004), we also tested the robustness of surrogates of multivariate patterns in community structure to temporal variation in community structure. Of the multivariate surrogate sets we examined, approaches that selected sets of taxa irrespective of their taxonomic affinities (e.g., Oliver and Beattie 1996, Fleishman et al. 2004), or which simply selected the most abundant taxa, performed consistently better than random selections of families and selection of a single higher taxon, provided that a sufficient number of families were identified $(>10 \%$ of all families). Our finding that using a single higher taxon as a surrogate of total biodiversity in marine commun- 


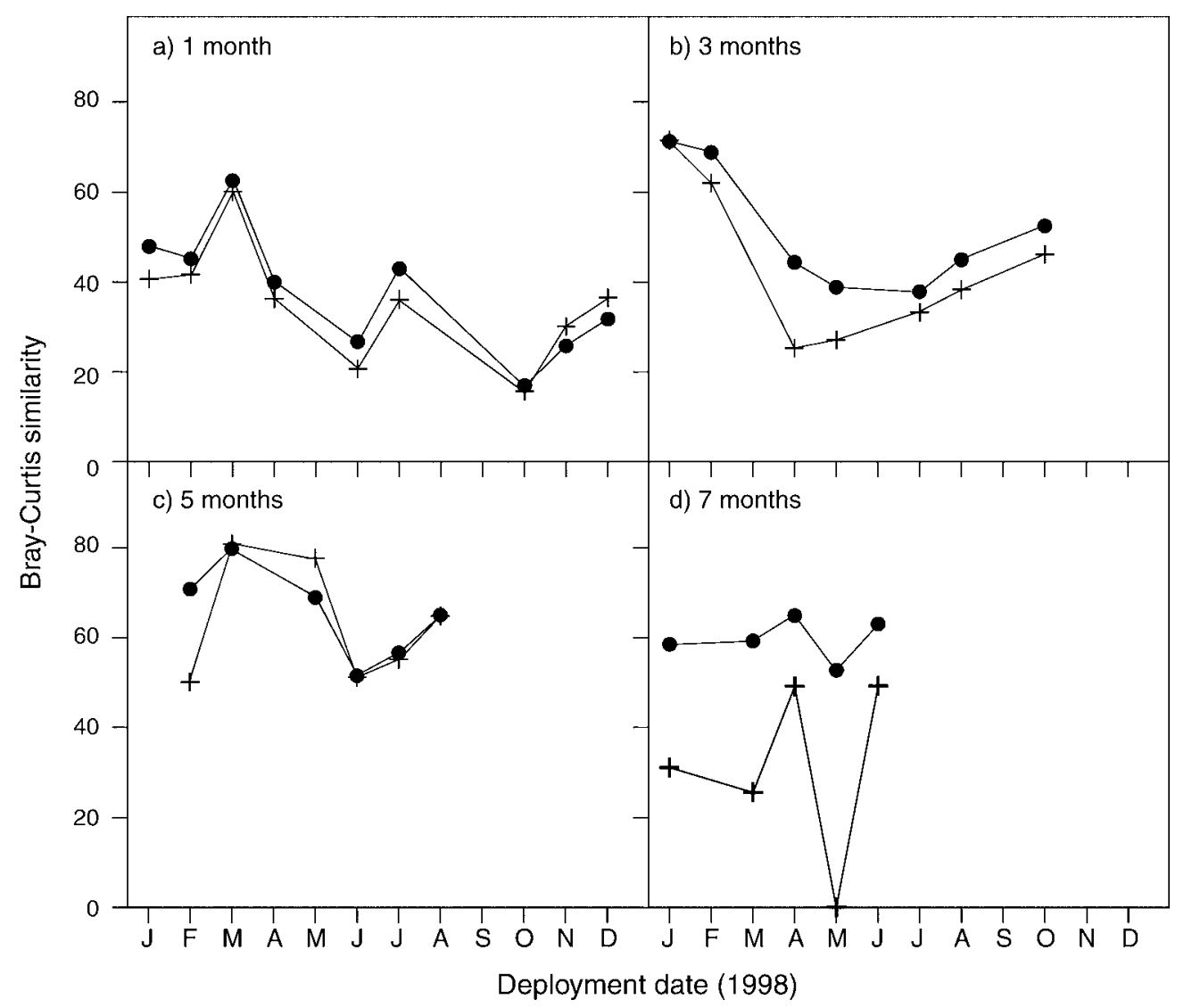

FIG. 5. Bray-Curtis similarity between the surrogate set identified by SIMPER (PRIMER5) for each deployment date and the surrogate set identified by SIMPER for holdfasts deployed in December 1997, for several representative community ages (1, 3, 5, and 7 months, panels a-d, respectively; see Appendix B for all community ages). Two techniques were used to select surrogates: $(+)$ taxa were included in a surrogate set if they contributed $\geq 5 \%$ to the average similarity within treatments and had a ratio of average similarity to standard deviation $\geq 1.4$; and $(\bullet)$ taxa were included in a surrogate set in order of decreasing percentage contribution to the average similarity within treatments until the cumulative contribution was $80 \%$ (note that this technique consistently selected a greater number of families in the surrogate set than the criteria based on contribution of $\geq 5 \%$ to average similarity).

ities is suboptimal is consistent with the results of Anderson et al. (2005b), but not of some others. In contrast with our conclusions, Gladstone (2002) suggested that marine reserves established to conserve mollusk diversity would also adequately protect total biodiversity (of noncryptic animals $>5 \mathrm{~mm}$ maximum dimension) on rocky shores in New South Wales, Australia, while Olsgard and Somerfield (2000) and Olsgard et al. (2003) showed that polychaetes are suitable surrogates of biodiversity in soft-sediment communities. These contrasting results probably reflect differences in the ecological roles of these higher taxa in different habitat types and highlight the importance of validating a surrogacy technique for the specific community being examined. We note however, that none of the above authors examined the performance of their surrogates across time intervals of different magnitude or season, nor did they compare the performance of their selected surrogates to other selections of taxa where taxonomic affinity was ignored.

\section{How should surrogates be identified?}

While the specific taxa selected as a surrogate suite will vary among different communities, and possibly even among similar communities in different environments, the techniques used to select taxa apply to any community type. Among the several techniques for selecting surrogate sets that did perform well, the choice of which to use will largely depend on the nature of the reference data used to select surrogates. Techniques that require information to compare between treatments (in our case, the different combinations of deployment date and community age) such as BVSTEP (Clarke and Warwick 2001) appear to be most useful. Under these circumstances the surrogate is selected because it correlates best with the change in overall community structure it is intended to detect. However, this approach requires access to pilot data collected across several treatments or through time.

When these kinds of pilot data are not available (for example, because of the expense of obtaining data), techniques that select taxa because they are abundant 
TABLE 2. Comparison of the similarity matrix contrasting communities defined by each combination of deployment date and community age based on all taxonomic groups with the equivalent similarity matrix based on each surrogate set.

\begin{tabular}{|c|c|c|c|}
\hline Surrogacy technique & $\begin{array}{l}\text { Reference } \\
\text { month }\end{array}$ & $\begin{array}{c}\text { No. taxa in } \\
\text { surrogate set }\end{array}$ & $\rho_{\mathrm{w}}$ \\
\hline BVSTEP & Mar & 20 & 95.32 \\
\hline BVSTEP & Feb & 20 & 94.92 \\
\hline BVSTEP & Jan & 20 & 94.19 \\
\hline Most abundant families & & 20 & 93.92 \\
\hline BVSTEP & Apr & 20 & 93.75 \\
\hline BVSTEP & Feb & 15 & 93.16 \\
\hline BVSTEP & Apr & 15 & 92.66 \\
\hline Most abundant families & & 15 & 92.56 \\
\hline BVSTEP & Jan & 15 & 92.38 \\
\hline SIMPER & Mar & 14 & 92.26 \\
\hline SIMPER & Apr & 13 & 92.12 \\
\hline BVSTEP & Apr & 12 & 91.43 \\
\hline SIMPER & Jan & 14 & 91.33 \\
\hline BVSTEP & Jan & 10 & 90.90 \\
\hline BVSTEP & Apr & 10 & 90.84 \\
\hline BVSTEP & Feb & 10 & 90.74 \\
\hline BVSTEP & Jan & 12 & 90.70 \\
\hline Most abundant families & & 12 & 90.54 \\
\hline BVSTEP & Mar & 15 & 90.37 \\
\hline BVSTEP & Feb & 12 & 90.19 \\
\hline BVSTEP & Mar & 12 & 89.40 \\
\hline Most abundant families & & 10 & 89.20 \\
\hline Random selection & & 20 & 88.89 \\
\hline Crustacea only & & 24 & 88.02 \\
\hline BVSTEP & Mar & 10 & 87.05 \\
\hline Most abundant families & & 5 & 86.73 \\
\hline BVSTEP & Apr & 5 & 86.64 \\
\hline SIMPER & Feb & 6 & 86.18 \\
\hline Mollusca only & & 32 & 85.82 \\
\hline Polychaeta only & & 21 & 85.45 \\
\hline BVSTEP & Jan & 5 & 85.31 \\
\hline BVSTEP & Feb & 5 & 85.30 \\
\hline Random selection & & 20 & 84.10 \\
\hline Random selection & & 20 & 83.06 \\
\hline Random selection & & 20 & 82.19 \\
\hline Random selection & & 20 & 77.63 \\
\hline Echinodermata only & & 12 & 77.05 \\
\hline BVSTEP & Mar & 5 & 76.41 \\
\hline
\end{tabular}

Notes: Reference month (where applicable) refers to the deployment date used to select a surrogate set. Comparisons of similarity matrices (based on Bray-Curtis distances of the fourth-root-transformed data) were made using the weighted Spearman rank correlation coefficient, $\rho_{\mathrm{w}}$; results are presented in descending order of goodness of fit (decreasing similarity). See Methods: Surrogacy analysis: Multivariate surrogates of community pattern for a description of the BVSTEP and SIMPER techniques.

and consistent can also be used (e.g., BVSTEP, SIMPER, or by selecting abundant taxa). However, in this case selection of surrogates should be confirmed subsequently since the composition of the surrogate set may vary with time. Encouragingly, our results showed that for macrofauna communities of kelp holdfasts, similar surrogate communities were identified (using SIMPER) for holdfast communities deployed on different dates and for different community ages, as long as a sufficient number of taxa were identified $(\sim 10 \%$ of the total) and the community was not at a very early stage of succession, particularly for deployments in winter months. Note that the performance of SIMPER was similar to that of using the most abundant families; however, we used SIMPER to select families that were both abundant and also consistently present in time. On this basis, using SIMPER to select surrogates should be preferred over selection of taxa based only on abundance.

\section{CONCLUSIONS}

Our results show that temporally robust surrogates can be identified; however, the nature of these surrogates will depend on how biodiversity is defined (Noss 1990), the type of community under study, and whether baseline data are available across several consecutive sampling

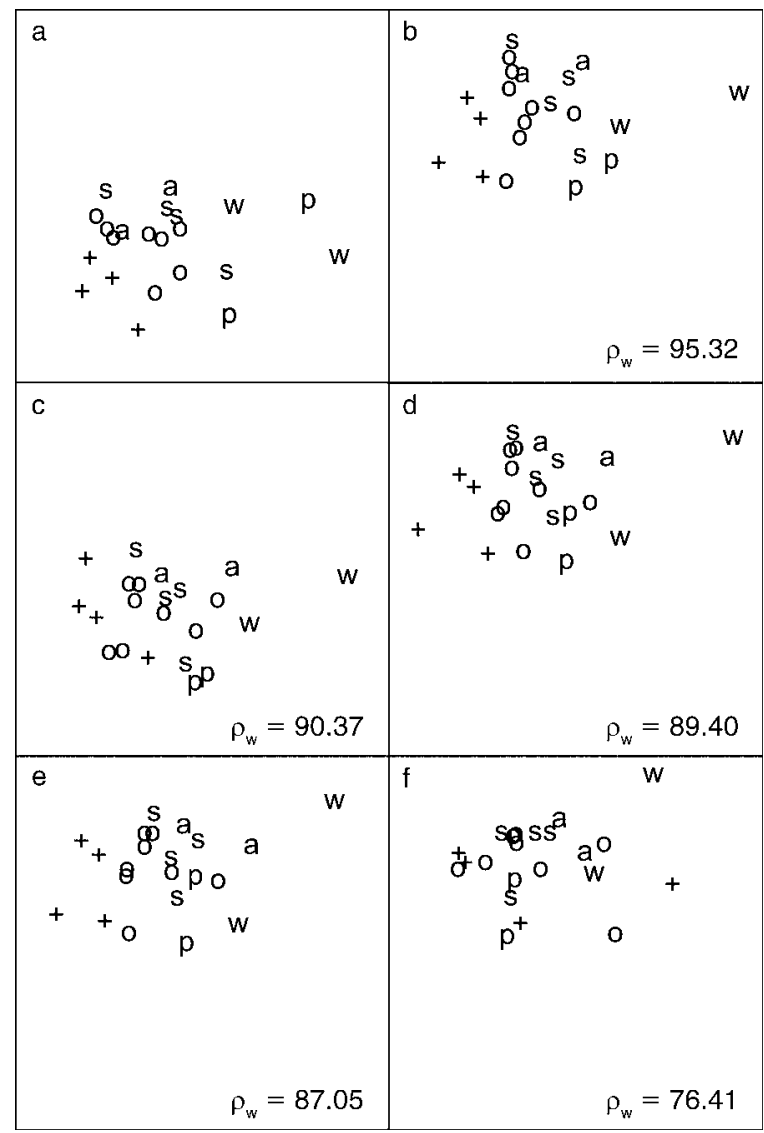

FIG. 6. NMDS (nonmetric multidirectional scaling) plots based on Bray-Curtis similarity (fourth-root-transformed data) for (a) the full data set including all families; and for various surrogate sets selected using BVSTEP (data from the deployment date March 1998), based on selections of (b) 20 families, (c) 15 families, (d) 12 families, (e) 10 families, and (f) five families. Although Bray-Curtis similarities were used to contrast all deployment dates and community ages, this figure presents only a subset of treatments to assist interpretation. For one-month-old communities, treatment symbols are s, deployed in December 1997, January 1998, February 1998, or December 1998; a, deployed in March or April 1998; w, deployed in June or July 1998; p, deployed in October or November 1998. All three-month-old communities are designated by "o," and all 10month-old communities by "+." In panels (b)-(f) we also report $\rho_{\mathrm{w}}$, the weighted Spearman rank correlation between the similarity matrix used to generate plot (a) and the similarity matrix used to generate each surrogate set. 
periods or from only a single sampling. Regardless of which surrogate is selected for study, the spatial and temporal robustness of the surrogate should be examined across relevant scales of observation. Where temporal data are not available, information on temporal variation might be gained by examining spatial variation in cases where the disturbance history of patches or lineal geographic features (e.g., coastlines) is known. Patches on a benthic "landscape" may be on an attractor describing oscillating community dynamics, but out of phase (Habeeb et al. 2005).

We note that the capacity to identify surrogates of kelp holdfast macrofauna communities that are temporally stable over 13 months does not mean that the surrogates will continue to be stable over longer time periods. Similarly, the effectiveness of a surrogate may differ before and after environmental impact. Environmental impact that disrupts community structure may change the relationship between a taxa and total biodiversity (e.g., Smith 1996, Olsgard and Somerfield 2000). Regular validation of a surrogate's performance is required throughout any monitoring program, particularly in the face of changing patterns of disturbance.

\section{ACKNOWLEDGMENTS}

This project was supported by funds from the School of Zoology and the Tasmanian Aquaculture and Fisheries Institute, University of Tasmania awarded to C. R. Johnson. Jeff Ross, Len Cusack, Dana Price, and Matt Field provided invaluable assistance in the field and laboratory. We thank two anonymous reviewers who provided useful criticism of the manuscript. This paper forms part of a Ph.D. for R. Magierowski who was supported by an Australian Postgraduate Award.

\section{Literature Cited}

Anderson, M. J., S. D. Connell, B. M. Gillanders, C. E. Diebel, W. M. Blom, J. E. Saunders, and T. J. Landers. 2005a. Relationships between taxonomic resolution and spatial scales of multivariate variation. Journal of Animal Ecology 74:636-646.

Anderson, M. J., C. E. Diebel, W. M. Blom, and T. J. Landers. 2005b. Consistency and variation in kelp holdfast assemblages: spatial patterns of biodiversity for the major phyla at different taxonomic resolutions. Journal of Experimental Marine Biology and Ecology 320:35-56.

Balmford, A., A. H. M. Jayasuriya, and M. J. B. Green. 1996. Using higher-taxon richness as a surrogate for species richness: II. local applications. Proceedings of the Royal Society of London B: Biological Sciences 263:1571-1575.

Barbour, M. T., J. L. Plafkin, B. P. Bradley, C. G. Graves, and R. W. Wisseman. 1992. Evaluation of EPA's rapid bioassessment benthic methods: metric redundancy and variability among reference stream sites. Environmental Toxicology and Chemistry 11:437-449.

Beccaloni, G. W., and K. J. Gaston. 1995. Predicting the species richness of Neotropical forest butterflies: Ithominae (Lepidoptera: Nymphalidae) as indicators. Biological Conservation 71:77-86.

Bray, J. R., and J. T. Curtis. 1957. An ordination of the upland forest communities of Wisconsin. Ecological Monographs 27: 325-349.

Cao, Y., A. W. Bark, and P. Williams. 1996. Measuring the responses of macroinvertebrate communities to water pollu- tion: a comparison of multivariate approaches, biotic and diversity indices. Hydrobiologia 341:1-19.

Chapman, A. R. O., and C. R. Johnson. 1990. Disturbance and organization of macroalgal assemblages in the northwest Atlantic. Hydrobiologia 192:77-121.

Clarke, K. R., and M. Ainsworth. 1993. A method for linking multivariate community structure to environmental variables. Marine Ecology Progress Series 92:205-219.

Clarke, K. R., and R. M. Warwick. 1998. Quantifying structural redundancy in ecological communities. Oecologia 113:278-289.

Clarke, K. R., and R. M. Warwick. 2001. Change in marine communities: an approach to statistical analysis and interpretation. Second edition. PRIMER-E, Plymouth, UK.

Colwell, R. K., and J. A. Coddington. 1994. Estimating terrestrial biodiversity through extrapolation. Philosophical Transactions of the Royal Society of London B 345:101-118.

Dahl, L., and K. Dahl. 2002. Temporal, spatial and substratedependent variations of Danish hard-bottom macrofauna. Helgoland Marine Research 56:159-168.

Daily, G. C., and P. R. Ehrlich. 1995. Preservation of biodiversity in small rainforest patches: rapid evaluations using butterfly trapping. Biodiversity and Conservation 4:3555.

Dayrat, B. 2005. Towards integrative taxonomy. Biological Journal of the Linnean Society 85:407-415.

Dayton, P. K., V. Currie, T. Gerrodette, B. D. Keller, R. Rosenthal, and D. Ven Tresca. 1984. Patch dynamics and stability of some California kelp communities. Ecological Monographs 54:253-289.

Dean, R. L., and J. H. Connell. 1987. Marine invertebrates in an algal succession. I. Variations in abundance and diversity with succession. Journal of Experimental Marine Biology and Ecology 109:195-215.

Diamond, J. M., M. T. Barbour, and J. B. Stribling. 1996. Characterising and comparing bioassessment methods and their results: a perspective. Journal of the North American Benthological Society 15:713-727.

Erdmann, M. V., and R. L. Caldwell. 1997. Stomatopod crustaceans as bioindicators of marine pollution stress on coral reefs. Proceedings of the Eighth International Coral Reef Syposium, Panama 2:1521-1526.

Faith, D. P., P. L. Dostine, and C. L. Humphrey. 1995. Detection of mining impacts on aquatic invertebrate communities: results of a disturbance experiment and the design of a multivariate BACIP monitoring programme at Coronation Hill, Northern Territory. Australian Journal of Ecology 20:167-180.

Fleishman, E., J. R. Thomson, R. MacNally, D. D. Murphy, and J. P. Pay. 2004. Using indicator species to predict species richness of multiple taxonomic groups. Conservation Biology 19:1125-1137.

Fore, L. S., J. R. Karr, and R. W. Wisseman. 1996. Assessing invertebrate responses to human activities: evaluating alternative approaches. Journal of the North American Benthological Society 15:212-231.

Franklin, J. F. 1988. Structural and functional diversity in temperate forests. Pages 166-175 in E. O. Wilson, editor. Biodiversity. National Academy Press, Washington, D.C., USA.

Garson, J., A. Aggarwal, and S. Sarkar. 2002. Birds as surrogates for biodiversity: an analysis of a data set from southern Quebec. Journal of Bioscience 27:347-360.

Gaston, K. J. 1996a. Biodiversity-congruence. Progress in Physical Geography 20:105-112.

Gaston, K. J. 1996b. Species richness: measure and measurement. Pages 77-113 in K. J. Gaston, editor. Biodiversity: a biology of numbers and difference. Blackwell Science, Oxford, UK.

Gaston, K. J., and T. M. Blackburn. 1995. Mapping biodiversity using surrogates for species richness: macro- 
scales and New World birds. Proceedings of the Royal Society of London B 262:335-341.

Gaston, K. J., and P. H. Williams. 1993. Mapping the world's species-the higher taxon approach. Biodiversity Letters 1:2-8.

Gladstone, W. 2002. The potential value of indicator groups in the selection of marine reserves. Biological Conservation 104: 211-220.

Glasby, T. M., and A. J. Underwood. 1998. Determining positions for control locations in environmental studies of estuarine marinas. Marine Ecology Progress Series 171:1-14.

Habeeb, R. L., J. Trebilco, S. Wotherspoon, and C. R. Johnson. 2005. Determining natural scales of ecological systems. Ecological Monographs 75:467-487.

Hammond, P. M. 1994. Practical approaches to the estimation of the extent of biodiversity in speciose groups. Philosophical Transactions of the Royal Society of London B 345:119-136.

James, R. J., M. P. Lincoln Smith, and P. G. Fairweather. 1995. Sieve mesh size and taxonomic resolution needed to describe natural spatial variation of marine macrofauna. Marine Ecology Progress Series 118:187-198.

Karr, J. R. 1981. Assessment of biotic integrity using fish communities. Fisheries 6:21-27.

Karr, J. R., P. R. Yant, and K. D. Fausch. 1987. Spatial and temporal variability of the index of biotic integrity in three midwestern streams. Transactions of the American Fisheries Society 116:1-11.

Kitching, R. L., D. Li, and N. E. Stork. 2001. Assessing biodiversity "sampling packages": how similar are arthropod assemblages in different tropical rainforests? Biodiversity and Conservation 10:793-813.

Kitching, R. L., A. G. Orr, L. Thalib, H. Mitchell, M. S. Hopkins, and A. W. Graham. 2000. Moss assemblages as indicators of environmental quality in remnants of upland Australia rain forest. Journal of Applied Ecology 37:284-297.

Kremen, C. 1992. Assessing the indicator properties of species assemblages for natural areas monitoring. Ecological Applications 2:203-217.

Lund, M. P., and C. Rahbek. 2002. Cross-taxon congruence in complementarity and conservation of temperate biodiversity. Animal Conservation 5:163-171.

McGeoch, M. A. 1998. The selection, testing and application of terrestrial insects as bioindicators. Biological Review 73:181201.

Mistri, M., and R. Rossi. 2001. Taxonomic sufficiency in lagoonal ecosystems. Journal of the Marine Biological Association of the UK 81:339-340.

Nandakumar, K. 1996. Importance of timing of panel exposure on the competitive outcome and succession of sessile organisms. Marine Ecology Progress Series 131:191-203.

Noss, R. F. 1990. Indicators for monitoring biodiversity: a hierarchical approach. Conservation Biology 4:355-364.

Oliver, I., and A. J. Beattie. 1996. Designing a cost-effective invertebrate survey: a test of methods for rapid assessment of biodiversity. Ecological Applications 6:594-607.

Olsgard, F., T. Brattegard, and T. Holthe. 2003. Polychaetes as surrogates for marine biodiversity: lower taxonomic resolution and indicator groups. Biodiversity and Conservation 12:1033-1049.
Olsgard, F., and P. J. Somerfield. 2000. Surrogates in marine benthic investigations - which taxonomic unit to target? Journal of Aquatic Ecosystem Stress and Recovery 7:25-42.

Olsgard, F., P. J. Somerfield, and M. R. Carr. 1997. Relationships between taxonomic resolution and data transformations in analyses of a macrobenthic community along an established pollution gradient. Marine Ecology Progress Series 149:173-181.

Pearson, D. L., and F. Cassola. 1992. World-wide species richness patterns of Tiger Beetles (Coleoptera: Cicindelidae): indicator taxon for biodiversity and conservation studies. Conservation Biology 6:376-391.

Samways, M. J., and N. S. Steytler. 1996. Dragonfly (Odonata) distribution patterns in urban and forest landscapes, and recommendations for riparian management. Biological Conservation 78:279-288.

Savage, A. A. 1982. Use of water boatmen (Corixidae) in the classification of lakes. Biological Conservation 23:55-70.

Schulze, C. H., M. Waltert, P. J. A. Kessler, R. Pitopang, Shahabuddin, D. Veddeler, M. Mühlenberg, S. R. Gradstein, C. Leuschner, I. Steffan-Dewenter, and T. Tscharntke. 2004 Biodiversity indicator groups of tropical land-use systems: comparing plants, birds, and insects. Ecological Applications 14:1321-1333.

Smith, S. D. A. 1996. The effects of domestic sewage effluent on marine communities at Coffs Harbour, New South Wales, Australia. Marine Pollution Bulletin 33:309-316.

Somerfield, P. J., and K. R. Clarke. 1995. Taxonomic levels, in marine community studies, revisited. Marine Ecology Progress Series 127:113-119.

Su, J. C., D. M. Debinski, M. E. Jakubauskas, and K. Kindscher. 2004. Beyond species richness: community similarity as a measure of cross-taxon congruence for coarse-filter conservation. Conservation Biology 18:167-173.

Underwood, A. J., and M. J. Anderson. 1994. Seasonal and temporal aspects of recruitment and succession in an intertidal estuarine fouling assemblage. Journal of the Marine Biological Association of the UK 74:563-584.

Underwood, A. J., and C. H. Peterson. 1988. Towards an ecological framework for investigating pollution. Marine Ecology Progress Series 46:227-234.

Valdecasas, A. G., and A. I. Camacho. 2003. Conservation to the rescue of taxonomy. Biodiversity and Conservation 12: $1113-1117$

Vane-Wright, R. I., C. J. Humphries, and P. H. Williams. 1991. What to protect? systematics and the agony of choice. Biological Conservation 55:235-254.

Warman, L. D., D. M. Forsyth, A. R. E. Sinclair, K. Freemark, H. D. Moore, T. W. Barrett, R. L. Pressey, and D. White. 2004. Species distributions, surrogacy, and important conservation regions in Canada. Ecology letters 7:374-379.

Wheeler, Q. D., P. H. Raven, and E. O. Wilson. 2004. Taxonomy: impediment or expedient? Science 303:285.

Williams, P. H., and K. J. Gaston. 1994. Measuring more of biodiversity: can higher-taxon richness predict wholesale species richness? Biological Conservation 67:211-217.

\section{APPENDIX A}

A figure showing Bray-Curtis similarity between the surrogate set identified by SIMPER (PRIMER5) for each community age and the surrogate set identified by SIMPER for one month holdfasts, for each deployment date (Ecological Archives A016-072-A1).

\section{APPENDIX B}

A figure showing Bray-Curtis similarity between the surrogate set identified by SIMPER (PRIMER5) for each deployment date and the surrogate set identified by SIMPER for holdfasts deployed in December 1997, for each community age (Ecological Archives A016-072-A2). 